\title{
Using Cartograms for Visualizing extended Floating Car Data (xFCD)
}

\author{
Christian Röger*, Jukka M. Krisp \\ University of Augsburg, Institute of Geography, Applied Geoinformatics, Alter Postweg 118, 86159 Augsburg, Germany \\ christian.roeger@geo.uni-augsburg.de \\ * Corresponding author
}

\begin{abstract}
This study assesses the usefulness of cartograms when visualizing extended Floating Car Data (xFCD). Cartograms deform regions in a map proportionally to assigned values. We apply this method for visualizing highresolution extended Floating Car Data (xFCD). Elaborating on this, we perform a case study in Mönchengladbach, Germany using 1.8 Million record points containing information about carbon dioxide (CO2) emissions based on an xFCD dataset. Utilizing a diffusion-based approach, we compute cartograms. Findings indicate a good suitability for identifying areas with a higher (or lower) average emission of $\mathrm{CO} 2$. We provide a documented workflow to compute cartograms based on parameters from an extended floating car dataset. The quality and spatial distribution of the basic dataset turns out to be important. Choosing the correct spatial subdivision of the research area as a basis for deforming areas is significant as it strongly influences the visual output.
\end{abstract}

Keywords: Cartograms, extended Floating Car Data (xFCD), floating car data (FCD), Visualization, spatial analysis

\section{Cartograms as a possibility for visualizing extended Floating Car Data (xFCD)}

Cartograms visualize data by deforming polygons based on their population value. Regions with a high values increase their size while areas with low values shrink. The popular website "worldmapper.org"" for example offers a number of cartograms. Figure 1 shows carbon dioxide (CO2) emissions from various countries throughout the world. Countries with high $\mathrm{CO} 2$ emissions are increased in their size, while countries with lower emissions decrease. This provides a map that distorts the way the world is usually seen.

In this paper, we state that cartograms can help visualizing high-resolution sensor data like extended Floating Car Data (xFCD). In xFCD, individual vehicles act as floating sensors recording information about the location of a car combined with parameters like fuel consumption and instantaneous carbon dioxide emission. By creating

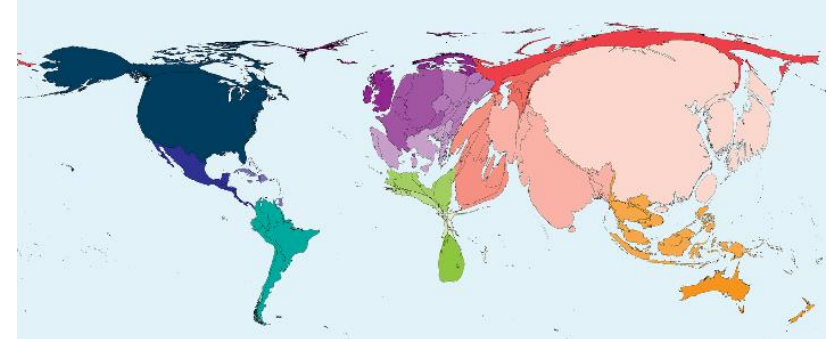

Figure 1. Cartogram of world carbon dioxide emissions in 2015. Source: www.worldmapper.org cartograms, we intend to explore extended Floating Car Data visually. Therefore, we investigate questions including:

- Which cartogram algorithms are commonly used? Which of them are applicable for our purpose?

- How can we pre-process our xFCD datasets in order to use them for cartogram generation?

- What are the benefits when visualizing xFCD using cartograms?

\subsection{Extended Floating Car Data (xFCD)}

Floating Car Data (FCD) is a possibility of gathering mobile traffic data for wide-area road networks. Therefore, single vehicles act as floating sensors. Historically, there have been other traffic data collection systems like inductive loops or video cameras. However, their characteristics as stationary systems with a need of installing on-road instruments has been leading to higher demands of FCD-based setups (Fabritiis et al. 2008). Generally, Floating Car Data consists of position (latitude, longitude, altitude) and timestamp information. Therefore, a GPS module is mandatory. Depending on the use case, Ranacher et al. (2016) propose an acquisition frequency of $1 / 2 \mathrm{~Hz}$ to $1 / 10 \mathrm{~Hz}$. Within a defined interval, records are acquired and transmitted to a central server.

\footnotetext{
${ }^{1}$ https://worldmapper.org/ (Accessed 2018/11/27)
} 
Extended Floating Car Data (xFCD) is an extension to common FCD. Therefore, original FCD records are enriched with information about internal and external phenomena of the vehicle or the environment. Extended data can include, for example, torque, $\mathrm{CO} 2$ emissions, fuel consumption, vehicle speed or driving direction. How can we connect the data collected by the sensors inside a car to a geographic position? The SAE J1962 specification provide a standardized hardware interface for recording incar sensor data. Since 1993, every European vehicle has an electronic system in order to control driving-specific functions like fuel injection, temperatures or appearing trouble codes. In 2001, this procedure has been standardized in the course of the Euro III agreement. For extracting data, an on-board diagnostic (OBD) connector can be used. When enriching this output with corresponding spatio-temporal data using an external device, extended Floating Car Data is being produced (Ortenzi et al. 2010).

Previous research has investigated the detection of critical situations or bottlenecks within road networks (Keler et al. 2017a), calculating travel times (Pfoser 2008, Rahmani et al. 2015), extracting traffic flow patterns (Jahnke et al. 2017, Keler et al. 2017b) and investigating environmental effects like carbon dioxide concentration or particulate matter emissions (Gühnemann et al. 2004, Röger et al. 2018).

In Germany, the enviroCar ${ }^{2}$ project has been initiated by a number of research partners to collect floating car data via a crowd-sourced approach. Individual drivers can contribute to the dataset by using a mobile application linked with an OBD Bluetooth adaptor. Resulting records can be accessed via an $\mathrm{API}^{3}$. Data is provided in a geoJSON format. There is a consistent data structure giving information about car specifications, driving velocities and board computer parameters. The enviroCar platform has recently been featured in the 'Grüne Welle' project $^{4}$.

The visualization of $\mathrm{xFCD}$ has been investigated by a number of researchers. Approaches include colorizing data points in various ways (Stanica et al. 2013). Andrienko and Andrienko (2007) propose binned views like heat maps or 2D histograms. Another approach by Cheng et al. (2013) focusses on visualizing data using road elements. Figure 2 illustrates these common visualization techniques for (extended) Floating Car Data.
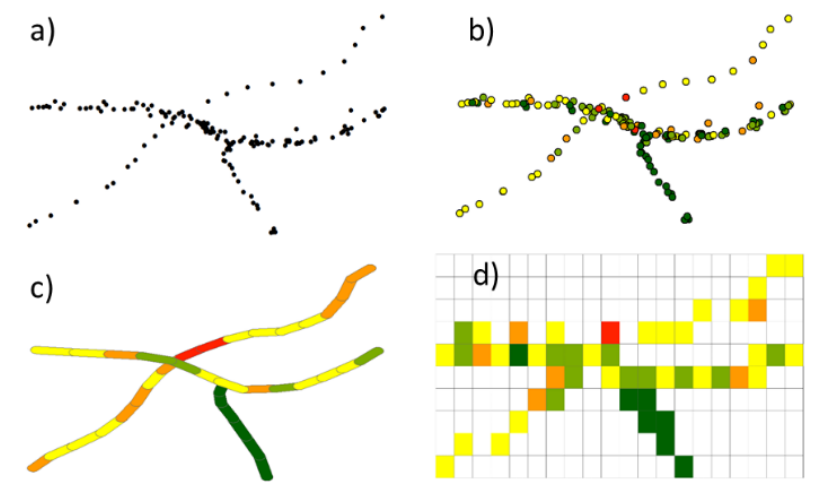

Figure 2. Visualization possibilities for extended Floating Car Data: original data (a), colorized data points (b), colorized road elements (c) and a binned visualization (d)

Figure 2 shows an initial data set (a) on the upper left hand side containing spatially distributed points attached with extended driving information. These extended values might contain information about the speed or the carbon dioxide emission of a vehicle. Subfigure (b) visualizes that additional information by colorizing the data points with a color grade from green (low values) to red (high values). The other two illustrations interpolate values by spatially joining data records to road trajectories (c) and a regular grid (d). Color grading gives information about the quantity of the calculated value.

\subsection{Cartograms}

Cartograms can be classified into area cartograms and distance based cartograms. The distance-based approach elaborates mainly on travel times or travel costs within a defined space. Deformation can be accomplished either from a defined center to other locations of a map or between each location of a region (Wang et al. 2017). Area cartograms base on population values given to polygons on a map. Common area based approaches simply scale regions so that they are proportional to their attached population areas. Additionally, continuous area cartograms keep the topology of a map so that neighboring regions stay neighbors (House and Kocmoud 1998). The key of creating an area-based cartogram is finding a transformation $r \rightarrow T(r)$ that converts space according to specified population values (Gastner and Newman 2004). Implementing an algorithm for finding a proper transformation has been a key question in cartogram related research. In an early approach, Tobler (1963) worked on deforming rectangular cells so that their area matches their population density value. The problem he faced was that his approach did not keep the map topology. In order to prevent overlapped or folded areas within a map, there had to be some additional constraints that made

\footnotetext{
https://envirocar.org (Accessed 2018/12/02)

https://envirocar.org/api (Accessed 2018/11/09)

4 https://www.moenchengladbach.de/de/rathaus/buergerinfo-az/planen-bauen-mobilitaet-umwelt-dezernat-

vi/fachbereich/strassenbau-und-verkehrstechnik-66/projektgruene-welle/ (Accessed 2018/11/07)
} 
the simple algorithm complex and slow (Gastner and Newman 2004). Dougenik et al. (1985) or Gusein-Zade and Tikunov (1993) elaborated on this problem. The key was to not only taking into account the individual population value of each cell but also computing interactions between cells when deforming them.

Gastner and Newman (2004) propose a diffusion-based approach. Their algorithm bases on the assumption that after calculating a cartogram, the population values of each cell get uniform. That is because population "flows away" from high-density fields to low-density fields. This process gets described by a density function $\rho(r)$. In this case, $r$ is a geographic position that is displaced according to the diffusion of the density. The displacement $r(t)$ of each point on a map can be calculated using the following formula (Gastner and Newman 2004):

$r(t)=r(0)+\int_{0}^{t} v\left(r, t^{\prime}\right) d t^{\prime}$

where $v\left(r, t^{\prime}\right)$ is the flowing velocity for a position $r$ at the time $t^{\prime}$. One disadvantage of the diffusion-based approach is that no-data fields get eliminated if their population is treated as zero. Gastner and Newman (2004) propose to use the mean population value instead in order to keep those areas "neutral" (Gastner and Newman 2004).

\section{A Case Study in Mönchengladbach, Germany}

For extended Floating Car Data, the area-based approach of computing a cartogram seems more applicable than the distance-based way. Keeping the topology is very important, to discover deformations in certain regions that would be hardly associable if they fold or overlap. Therefore, we apply the diffusion-based approach by Gastner and Newman (2004).

For the case study, we use the enviroCar database. As a study area, we choose the district of Mönchengladbach in Germany. In 2016, the project 'Grüne Welle' encouraged citizens of the town to contribute $\mathrm{XFCD}$ in order to improve the traffic flow of the city ${ }^{5}$. Hence, there is a disproportionately high count of record points within the enviroCar dataset located in the district of Mönchengladbach.

We use the enviroCar API for accessing the dataset. After downloading, we spatially clip the data for selecting only the points within the Mönchengladbach district. The dataset contains a total amount of 2349383 records. Table 1 shows the data schema, which contains several fields filled with specific driving data. The fields car_ID and track_ID give information about a specific car driving on a specific trajectory. Extended driving information includes fueltype, speed, consumption and instantaneous_co2. The carbon dioxide field describes the instantaneous $\mathrm{CO} 2$ emission of a car in kilograms per hour.

\begin{tabular}{|l|l|}
\hline Data field & Example \\
\hline object_ID & 2443896 \\
Latitude & 51.1950749861 \\
Longitude & 6.44354096668 \\
car_ID & $574 \mathrm{e} 78 \mathrm{cbe} 4 \mathrm{~b} 09078 f 97 \mathrm{bbb} 4 \mathrm{a}$ \\
track_ID & 5accf74d44ea8508c538ce15 \\
Datetime & 2018-03-05T20:04:36Z \\
Fueltype & Gasoline \\
Speed & 38.6349726915 \\
Consumption & 2.56207163322 \\
instantaneous_co2 & 6.02086833808 \\
\hline
\end{tabular}

Table 1. Data schema and example entry for the resulting dataset

Outliers and records without $\mathrm{CO} 2$ emission values are removed from the initial dataset. This process results in a dataset with 1798446 record points. As a next step, the borders used for the cartogram are defined. We define a grid of $2 \mathrm{~km} * 2 \mathrm{~km}$ for the study area. For a better orientation, we include major roads as well as cities with a population value higher than 10000 . Within the study area, there are a number of highways ('Autobahn' and 'Bundesstraße'), primary/secondary roads ('other major roads') and three towns (Mönchengladbach, Rheydt and Rheindahlen). The data origins from the OpenStreetMap $(\mathrm{OSM})^{6}$ project.

The distribution of data points within the data set is uneven. There are regions with a higher point density and regions with a lower density of record points. Figure 3 shows the study area and a classified grid based on the number of record points within each grid cell.

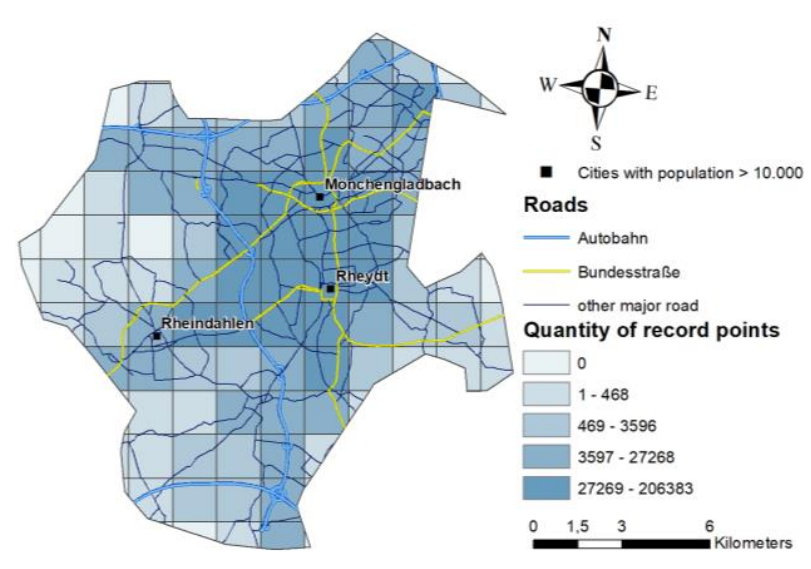

Figure 3. Map of the study area showing the count of record points per grid cell

\footnotetext{
5 https://www.moenchengladbach.de/de/rathaus/buergerinfo-az/planen-bauen-mobilitaet-umwelt-dezernat-

vi/fachbereich/strassenbau-und-verkehrstechnik-66/projektgruene-welle/ (Accessed 2018/11/07) 
The major roads are symbolized using bold blue lines ('Autobahn'), yellow lines ('Bundesstraße') and narrow dark blue lines ('other major roads'). Three cities with a population value of over 10000 are displayed using a black square. The regular grid is visualized using boxes with a black frame. The grid cells are enriched with the quantity of record points within each cell. That information is displayed using a color gradient from light blue (no record point) to dark blue (up to 206383 record points). The areas surrounding the three cities Mönchengladbach, Rheindahlen and Rheydt as well as areas around the Autobahn seem to have a higher count of record points than the rest of the study area. Additionally, there are twenty grid cells with no record point, including mainly regions at the border of the study area. It remains open how to deal with cells that contain a proportionally low quantity of points. Record points within a grid cell with a low point count could be outliers or not representable for the dataset as a whole. Gastner-Newman Cartograms expect empty polygons to have a population value that is equal to the mean population of each polygon (Gastner and Newman 2004). Consequently, we compute the mean CO2 value of all grids and assign it to the cells with no data. The initial map after pre-processing is displayed in Figure 4.

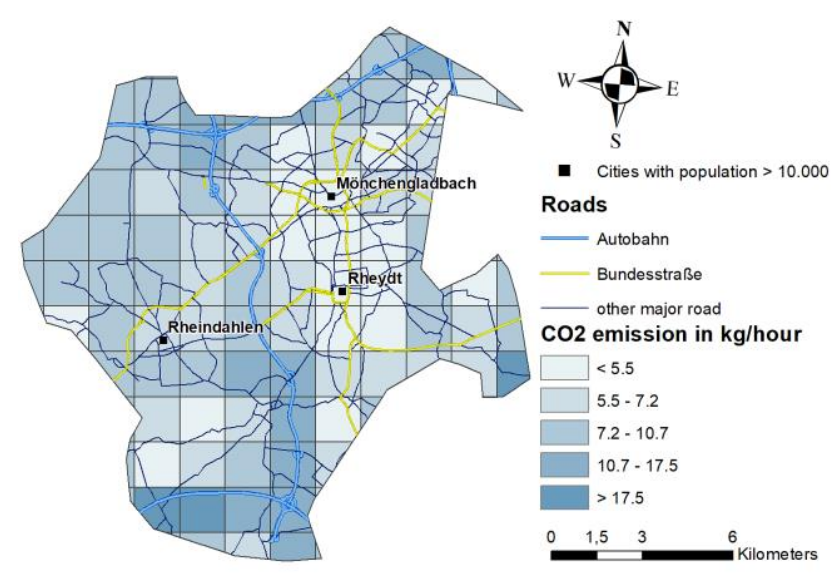

Figure 4. Initial map of the research area showing mean $\mathrm{CO} 2$ emission values per grid cell, major roads and main cities

Figure 4 shows the mean carbon dioxide emission values. They are displayed using a color gradient from light blue (low value) to dark blue (high value). There are grid cells with some higher values noticeable next to Autobahn roads. Especially next to the Autobahn interchange in the south of the study area, there seems to be an aggregation of higher $\mathrm{CO} 2$ emission values. The main cities are mainly surrounded by grid cells with comparatively low mean carbon dioxide values, especially around Mönchengladbach and Rheydt.

After creating the initial map, we calculate the cartogram shown in Figure 5.

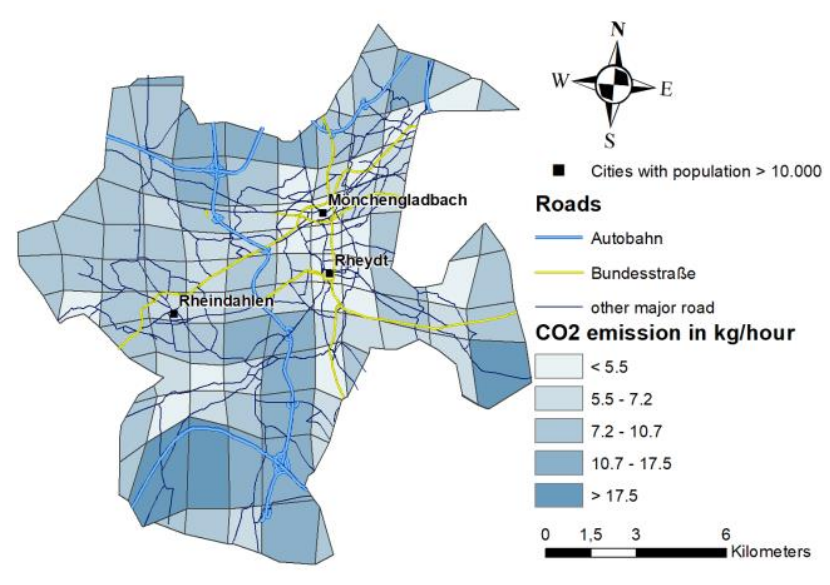

Figure 5. Cartogram of the study area showing enlarged grid cells (high mean $\mathrm{CO} 2$ values) and shrinked grid cells (low mean $\mathrm{CO} 2$ values)

Figure 5 depicts the mean carbon dioxide emission value of each cell which is used as a basis for deforming the grid cells. Regions next to the Autobahn have higher mean CO2 emission by individual cars indicated by bigger grid cells. Since the areas next to the three main cities have smaller grid cells than the average, there might be a lower mean carbon dioxide emission. The biggest cells accumulate in the south of the study area, next to the Autobahn interchange. A remarkable concentration of smaller cells can be found around Mönchengladbach and Rheydt.

The data provides the possibility to investigate the emission values for the different seasons of a year. Before joining, points are separated into spring, summer, autumn and winter by using the date/time field of the enviroCar dataset. This process results in a dataset with 497294 record points for winter, 916044 record points for summer, 146970 record points for autumn and 70171 record points for the season of winter.

Figure 6 shows four different cartograms. The grid cells are distorted according to the average $\mathrm{CO} 2$ emissions within these areas for the particular season. There is an indication for significant changes throughout the four seasons. The cartogram for spring has a similar appearance as the cartogram for the whole year (depicted in Figure 5), meaning that there are smaller cells next to the cities and bigger cells next to the Autobahn. In summer, there appears to be a similar situation. However, the mean carbon dioxide emission values seem to be smaller in the environment of the cities of Mönchengladbach and Rheydt. In autumn, the number of cells with a darker blue color show an increase. Even in the cities, there is an indication that the average instantaneous carbon dioxide emission per car rises compared to spring and summer. The cartogram showing the situation for winter shows darker cells than in the other seasons. This indicates the highest carbon dioxide emissions of all seasons in winter. Additionally we compute basic statistics of the dataset for the four seasons of a year shown in table 2 . 


\begin{tabular}{|l|l|l|l|l|}
\hline & Spring & Summer & Autumn & Winter \\
\cline { 1 - 1 } Mean & 8.35 & 8.26 & 10.04 & 10.95 \\
\cline { 1 - 1 } Min & 4.71 & 3.27 & 4.40 & 3.90 \\
\cline { 1 - 1 } & 26.00 & 24.72 & 26.52 & 26.05 \\
\cline { 1 - 1 } St. dev & 3.53 & 3.64 & 4.11 & 4.46 \\
\hline
\end{tabular}

Table 2. Statistics for the cartograms shown in Figure 6 giving information about mean, minimum, maximum and standard deviation values for the average $\mathrm{CO} 2$ emission per vehicle

Table 2 lists selected statistical variables (mean, minimum, maximum and standard deviation) for the dataset that provides the basis for the cartograms in Figure 6. In spring and summer seasons, there are similar mean and standard deviation values, meaning the carbon dioxide emission is analogical within the two seasons. This fact is also noticeable looking at the cartograms. In addition, autumn and winter seasons have the highest mean carbon dioxide emission. There is also a strong indication for that when comparing the cartograms in Figure 6. As stated, the cartogram for the winter season seems to provide the highest mean carbon dioxide emission values per vehicle.

\section{Discussion and Conclusions}

The case study shows that the diffusion-based approach by Gastner and Newman (2004) is an applicable way of producing cartograms. We also show that extended Floating Car Data (xFCD) is a capable source for enriching geographic regions with high-resolution sensor data.

The point density within individual grid cells matters. The distribution of record points is uneven throughout the study area. Dealing with empty grid cells needs to be considered, as Gastner and Newman (2004) propose to assign the mean value of all cells to them. This makes them 'neutral'. However, it is questionable how to deal with cells that include a disproportionally low count of record points since there is the chance of outliers or unrepresentative values to have an influence on the resulting cartogram.

Apart of the quantity, the quality of each $\mathrm{xFCD}$ record point is also a factor for calculating the population values for a cartogram. Some of the enviroCar record points do not have emission values attached, so they are not relevant for the computation of the area population. Furthermore, the calculation of the carbon dioxide emission directly relies on parameters of the on-board computer of a car. It is not documented how precise those values are depending
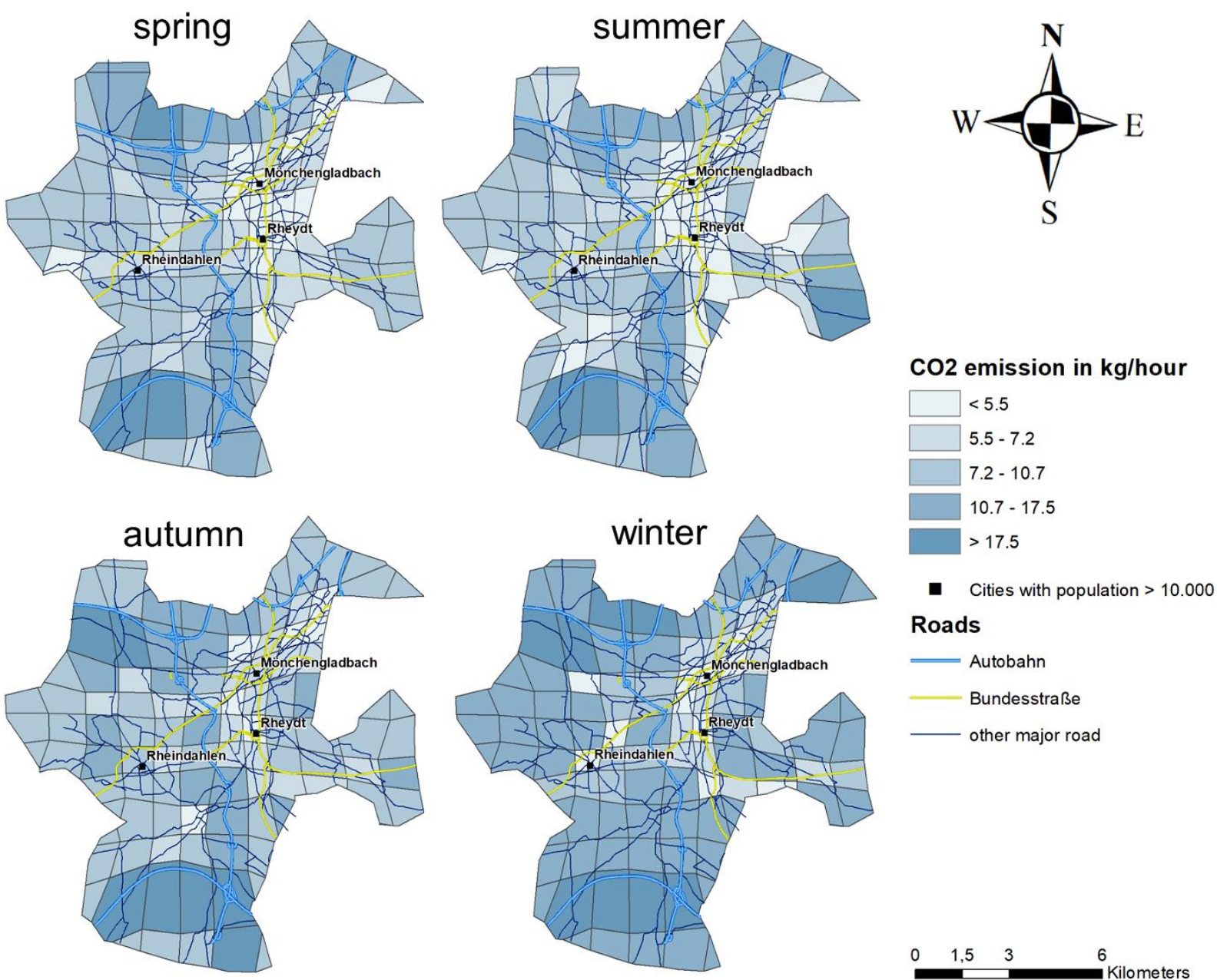

Roads

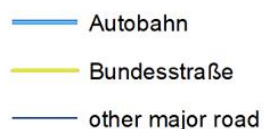

- other major road

Figure 6. Cartograms of four seasons (spring, summer, autumn, winter) showing areas with higher $\mathrm{CO} 2$ emission values and regions with lower $\mathrm{CO} 2$ emission values 
on the car manufacturer. How to treat cells with a varying quality of record points stays an open research question.

Another discussable point is the choice of the subdivision of a study area. We chose a regular grid of $2 \mathrm{~km} * 2 \mathrm{~km}$ cells. The size of the grid cells as well as the placement of them within the study area directly affects the appearance of the resulting cartogram. In our case study, dealing with several grid sizes would possibly have been beneficial. In addition, the factor of generalization increases as the size of the individual cells within a grid expands. It is also possible to use administrative borders instead of regular grid cells as suggested by Gusein-Zade and Tikunov (1993) or Gastner and Newman (2004).

Concluding this work, the quality of the results mainly depends on three factors: (1) the quality and spatial distribution of the basic dataset, (2) the choice of cell types and cell sizes and (3) the choice of the cartogram transformation algorithm.

Concerning the quality of the dataset, there have to be enough data points in total for a heterogeneous result and enough data points per cell so that there is a good distribution throughout the study area. In addition, the data source has to be reliable so that the quality of the dataset is acceptable.

Choosing the right spatial division is an important factor when creating cartograms. There is the possibility of defining a regular grid or using administrative borders. The choice of the grid size directly influences the resulting cartogram. In the end, deformation within a map should be clearly visible and explainable.

Selecting the correct cartogram algorithm is significant. Since we work with spatially distributed data points, we choose an area based cartogram approach. For the resulting map, using an algorithm that keeps the topology is important in order to keep the grid cells associable. The diffusion-based algorithm of Gastner and Newman (2004) is a good choice for creating cartograms based on highresolution sensor data.

An open research question is how to deal with cells that deform based on a low count of record points compared to other cells. In our case study, we discover that our data produces areas with computed population values based on a low quantity of record points. It has to be taken into account that the influence of outliers or non-representative points is higher in grid cells with a smaller amount of record points as a data basis.

Concerning future research, we suggest to investigate preprocessing extended Floating Car Data (xFCD) in order to produce a higher precision for the population values as a basis for calculating cartograms. Also considering the density of record points on certain roads will be an important task. Doing so, examining other datasets in different study areas will be a challenge. Experimenting with different cell sizes will also be part of the future work. Another task will be to test more algorithms for creating cartograms. Using distance-based approaches utilizing the carbon dioxide value as cost seems to be promising.

\section{References}

Andrienko, N. and Andrienko, G. (2007). Designing visual analytics methods for massive collections of movement data. Cartographica: The International Journal for Geographic Information and Geovisualization, 42(2), 117-138.

Cheng, T., Tanaksaranond, G., Brunsdon, C. and Haworth, J. (2013). Exploratory visualisation of congestion evolutions on urban transport networks. Transportation Research Part C: Emerging Technologies, 36, 296-306.

Dougenik, J. A., Chrisman, N. R., \& Niemeyer, D. R. (1985). An algorithm to construct continuous area cartograms. The Professional Geographer, 37(1), 75-81.

Fabritiis, C., Ragona, R. and Valenti, G. (2008). Traffic estimation and prediction based on real time floating car data. In: Intelligent Transportation Systems, 2008. ITSC 2008. 11th International IEEE Conference on. IEEE, S. 197-203.

Gastner, M. T. and Newman, M. E. (2004). Diffusionbased method for producing density-equalizing maps. Proceedings of the National Academy of Sciences, 101(20), 7499-7504.

Gühnemann, A., Schäfer, R. P., Thiessenhusen, K. U. and Wagner, P. (2004). Monitoring traffic and emissions by floating car data. Institute of Transport Studies Working Paper, (ITS-WP-04-07).

Gusein-Zade, S. M., \& Tikunov, V. S. (1993). A new technique for constructing continuous cartograms. Cartography and Geographic Information Systems, 20(3), 167-173.

House, D. H. and Kocmoud, C. J. (1998). Continuous cartogram construction. In Proceedings of the conference on Visualization'98 (pp. 197-204). IEEE Computer Society Press.

Jahnke, M., Ding, L., Karja, K. and Wang, S. (2017). Identifying origin/destination hotspots in floating car data for visual analysis of traveling behavior. In: Progress in Location-Based Services 2016: Springer, S. 253-269.

Keler, A., Krisp, J. M., Ding, L. (2017a). Detecting vehicle traffic patterns in urban environments using taxi trajectory intersection points. In: Geo-spatial Information Science 20 (4), S. 333-344.

Keler, A., Krisp, J. M. and Ding, L. (2017b). Visualization of Traffic Bottlenecks: Combining Traffic Congestion with Complicated Crossings. In: International Cartographic Conference. Springer, S. 493-505.

Ortenzi, F. and Costagliola, M. A. (2010). A new method to calculate instantaneous vehicle emissions using OBD data (No. 2010-01-1289). SAE Technical Paper.

Pfoser, D. (2008). Floating car data. In: Encyclopedia of GIS: Springer, S. 321.

Rahmani, M., Jenelius, E. and Koutsopoulos, H. N. (2015). Non-parametric estimation of route travel time distributions from low-frequency floating car data. In: Transportation Research Part C: Emerging Technologies 58, S. 343-362. 
Ranacher, P., Brunauer, R., van der Spek, S. and Reich, S. (2016). What is an appropriate temporal sampling rate to record floating car data with a GPS? In: ISPRS International Journal of Geo-Information 5 (1), S. 1.

Röger, C., Keler, A. and Krisp, J. M. (2018). Examining the Influence of Road Slope on Carbon Dioxide Emission using Extended Floating Car Data. In: Adjunct Proceedings of the 14th International Conference on Location Based Services. ETH Zurich, S. 135-140.

Stanica, R., Fiore, M. and Malandrino, F. (2013). Offloading floating car data. In World of Wireless, Mobile and Multimedia Networks (WoWMoM), 2013 IEEE 14th International Symposium and Workshops on a (pp. 1-9). IEEE.

Tobler, W. R. (1963). Geographic area and map projections. Geographical review, 53(1), 59-78.

Wang L., Ding L., Krisp J.M. and Li X.: (2018) Design and Implementation of Travel-time Cartograms. KN Kartographische Nachrichten, Journal of Cartography and Geographic Information (1), p. 13-20 